\title{
Evaluation of the GenoType Mycobacteria Direct assay for the simultaneous detection of the Mycobacterium tuberculosis complex and four atypical mycobacterial species in smear-positive respiratory specimens
}

\author{
A.-Louise Seagar, ${ }^{1}$ Carmel Prendergast, ${ }^{2}$ F. Xavier Emmanuel, ${ }^{1}$ \\ Alan Rayner, ${ }^{1}$ Susan Thomson $^{3}$ and lan F. Laurenson ${ }^{1}$ \\ ${ }^{1}$ Scottish Mycobacteria Reference Laboratory, Department of Laboratory Medicine, Royal Infirmary \\ of Edinburgh, 51 Little France Crescent, Edinburgh EH16 4SA, UK \\ ${ }^{2}$ Microbiology Department, Royal Brompton Hospital, Sydney Street, London SW3 6NP, UK \\ ${ }^{3}$ Mast Group Ltd, MAST House, Derby Road, Bootle, Merseyside L20 1EA, UK
}

Correspondence

A.-Louise Seagar

Louise.Seagar@luht.scot.nhs.uk

Received 29 June 2007

Accepted 2 February 2008

\begin{abstract}
A novel, commercially available reverse hybridization assay [GenoType Mycobacteria Direct (GTMD), version 2.0; Hain Lifescience] was evaluated for the direct detection of five clinically relevant mycobacterial species [Mycobacterium tuberculosis complex (MTBC), Mycobacterium avium, Mycobacterium malmoense, Mycobacterium kansasii and Mycobacterium intracellulare] from 54 smear-positive respiratory specimens and the findings were compared with culture results. Three approaches were used for specimen preparation using either whole or 'split' sample volumes and $\mathrm{N}$-acetyl-L-cysteine/3 $\% \mathrm{NaOH}$ or $4 \% \mathrm{NaOH}$ as decontamination chemicals.

Forty-three out of 52 samples in which RNA amplification was successful gave GTMD results that concurred with the identification of the cultured isolate. All cases of MTBC were detected. Twenty-two samples contained M. tuberculosis complex, seven had M. kansasii, four had $M$. malmoense, seven contained atypical mycobacteria other than those detectable using the GTMD assay and three specimens contained no viable mycobacteria. The assay is easy to use and can be completed in one working day. Results interpretation is facilitated by the inclusion of an internal amplification control with each sample to allow identification of specimens containing amplification inhibitors. A positive GTMD result will quickly identify patients with MTBC infection or provide specific identification of four other atypical mycobacteria from the same specimen. This allows more rapid drug susceptibility testing, treatment, and public health and infection control decisions.
\end{abstract}

\section{INTRODUCTION}

Tuberculosis (TB) is caused by Mycobacterium tuberculosis and globally accounts for 2 million deaths annually. The incidence of TB is around 7.5 per 100000 of the population in Scotland (Shakir et al., 2007) and 14.0 per 100000 in the UK (Health Protection Agency Centre for Infections, 2007). Increasingly, non-tuberculous mycobacteria (NTM), notably Mycobacterium avium, Mycobacterium intracellulare, Mycobacterium malmoense and Mycobacterium kansasii, have been implicated in opportunistic infections, particularly in

Abbreviations: AFB, acid-fast bacilli; CDAT, commercial direct amplification test; GTMD, GenoType Mycobacteria Direct; MTBC, Mycobacterium tuberculosis complex; NTM, non-tuberculous mycobacteria; SMRL, Scottish Mycobacteria Reference Laboratory; TB, tuberculosis. immunocompromised patients and in patients with chronic lung diseases. However, their epidemiology remains poorly understood (Falkinham, 1996). It is important to differentiate quickly between $M$. tuberculosis complex (MTBC) species and NTM, as this enables earlier decisions on optimum drug therapy and appropriate infection control measures. In addition, using molecular identification methods on primary samples to differentiate between MTBC and the different NTM species may enable a better understanding of the epidemiology of this complex group of organisms and their relevance in human disease.

Historically, laboratory diagnosis of MTBC infection has relied heavily on the time-consuming process of culturing organisms from clinical specimens using solid or liquid media. The advent of fast, reliable and sensitive molecular techniques for the direct detection of MTBC in 
smear-positive respiratory samples has the potential to revolutionize laboratory and clinical practice. Indications for their use in clinical samples are now included in British tuberculosis guidelines (National Collaborating Centre for Chronic Conditions, 2006). Several commercial direct amplification tests (CDATs) have been developed to allow rapid detection of mycobacteria in pulmonary and extrapulmonary specimens. Such assays work on the basic principle of nucleic acid amplification, although different enzymes and strategies are employed (Table 1). Species identification is usually limited to MTBC organisms, and an internal amplification control to monitor amplification inhibition is not always included. Nonetheless, they generally have good specificity and sensitivity for smearpositive respiratory specimens when compared with culture and clinical diagnosis and have the potential to substantially reduce the time taken to diagnosis. Non-sputum samples such as pleural fluids, cerebrospinal fluids and smearnegative samples are associated with lower test sensitivities (Piersimoni \& Scarparo, 2003).

The study described here evaluated the GenoType Mycobacteria Direct (GTMD) assay, version 2.0 (Hain Lifescience), which is designed to detect five clinically relevant mycobacterial species (MTBC, M. avium, $M$. malmoense, M. kansasii and M. intracellulare) directly from respiratory specimens following decontamination.

\section{METHODS}

Respiratory samples. The 51 sputum and 3 bronchoalveolar lavage specimens included in the study were received at the Scottish Mycobacteria Reference Laboratory (SMRL; $n=39$ ) or the Royal
Brompton Hospital $(n=15)$ for the detection of acid-fast bacilli (AFB) using routine microscopy and culture methods.

Microscopic examination. A loopful of each specimen was placed directly on a microscope slide and stained with auramine phenol using standard procedures. Slides were examined at $\times 400$ magnification. The number of AFB present was recorded as $+(1-10$ bacilli in ten fields), $++(1-10$ bacilli per field $)$ or $+++(10$ or more bacilli per field).

\section{Specimen processing and decontamination}

In some cases, referred specimens were of small volume $(<5 \mathrm{ml})$ so there was limited material available for additional tests once a sufficient volume had been allocated for routine tests. To assess the effect of specimen volume and the choice of decontamination method on test performance, three different approaches were used for specimen preparation using two different decontamination chemicals.

Group A: N-acetyl-L-cysteine (NALC)-treated 'split' specimens. After direct microscopy had been performed at the SMRL, 22 samples containing sufficient volume were 'split' into two equal aliquots of at least $1 \mathrm{ml}$ each and decontaminated using NALC/3\% $\mathrm{NaOH}$ (referred to as the NALC method). One aliquot was processed for routine mycobacterial culture using solid egg medium (two modified Löwenstein-Jensen slopes, one containing glycerol and one containing pyruvate as growth supplements) and rapid liquid culture (Mycobacterial Growth Indicator Tube; Becton Dickinson). The other aliquot was either decontaminated and tested immediately using the GTMD test $(n=2)$ or the respiratory specimen was stored at $-70{ }^{\circ} \mathrm{C}(n=20)$ for no longer than 7 months (mean 2.75 months). NALC decontamination was performed on the stored specimen and the resulting pellet was resuspended in $0.5 \mathrm{ml}$ phosphate buffer before starting the cell-disruption stage of the GTMD test.

Group B: NALC-treated 'whole' specimens. The number of specimens that could be collected in group A was limited, as only those where excess specimen material was available at SMRL could be

Table 1. Comparison of currently available CDATs

IAC, internal amplification control; NASBA, nucleic acid sequence-based amplification; BAL, bronchoalveolar lavage; CSF, cerebrospinal fluid.

\begin{tabular}{|c|c|c|c|c|c|c|}
\hline Molecular test & Supplier & Technology & Target sequence & IAC & Species detected & Specimen type \\
\hline $\begin{array}{l}\text { GenoType } \\
\text { Mycobacteria Direct }\end{array}$ & Hain Lifescience & $\begin{array}{c}\text { NASBA + reverse } \\
\text { hybridization }\end{array}$ & $23 \mathrm{~S}$ rRNA & Yes & $\begin{array}{l}\text { MTBC, M. avium, } \\
\text { M. intracellulare, } \\
\text { M. kansasii, M. } \\
\text { malmoense }\end{array}$ & $\begin{array}{l}\text { Pulmonary and } \\
\text { extrapulmonary } \\
\text { specimens (except } \\
\text { for blood) }\end{array}$ \\
\hline $\begin{array}{l}\text { GenoType } \\
\text { MTBDRplus }\end{array}$ & Hain Lifescience & $\begin{array}{l}\text { Multiplex PCR + reverse } \\
\text { hybridization }\end{array}$ & $\begin{array}{l}\text { rpoB, kat } G \text { and } \\
\text { inhA genes }\end{array}$ & Yes & $\begin{array}{l}\text { MTBC (with } \\
\text { rifampicin and } \\
\text { isoniazid resistance) }\end{array}$ & $\begin{array}{l}\text { Smear-positive } \\
\text { pulmonary } \\
\text { specimens }\end{array}$ \\
\hline Amplified MTD & Gen-Probe & $\begin{array}{l}\text { Transcription-mediated } \\
\text { amplification }\end{array}$ & 16S rRNA & No & MTBC & $\begin{array}{l}\text { Smear-positive and } \\
\text { smear-negative } \\
\text { respiratory samples }\end{array}$ \\
\hline $\begin{array}{l}\text { Cobas Amplicor } \\
\text { MTB }\end{array}$ & $\begin{array}{l}\text { Roche Molecular } \\
\text { Systems }\end{array}$ & Automated PCR & 16S DNA & Yes & MTBC & $\begin{array}{l}\text { Smear-positive } \\
\text { pulmonary } \\
\text { specimens }\end{array}$ \\
\hline INNO-LiPA Rif. TB & Innogenetics & $\begin{array}{l}\text { Nested PCR + line } \\
\text { probe assay }\end{array}$ & rpoB gene & No & $\begin{array}{l}\text { MTBC (and } \\
\text { rifampicin } \\
\text { resistance) }\end{array}$ & Sputum, biopsies \\
\hline $\begin{array}{l}\text { Artus Mycobac. diff. } \\
\text { PCR kit CE }\end{array}$ & Qiagen & Real-time PCR & $\begin{array}{l}\text { Mycobacterial } \\
\text { nitrate reductase } \\
\text { promoter }\end{array}$ & Yes & $\begin{array}{l}\text { MTBC and } \\
\text { M. avium complex }\end{array}$ & $\begin{array}{l}\text { Sputum; BAL; CSF; } \\
\text { bronchial secretion }\end{array}$ \\
\hline
\end{tabular}


divided. Therefore, for 17 sputum samples, the entire specimen volume was decontaminated using the NALC method. The resulting pellet was then resuspended in $1.5 \mathrm{ml}$ phosphate buffer. One millilitre was used to inoculate solid and liquid culture media (as outlined above) and the remaining $0.5 \mathrm{ml}$ volume was stored at $4{ }^{\circ} \mathrm{C}$ (for no longer than 3 days).

Group C: NaOH-treated 'whole' specimens. A modified Petroff's method using $4 \% \mathrm{NaOH}$ (referred to as the $\mathrm{NaOH}$ method) was used at the Royal Brompton Hospital for decontamination of the whole volume from 15 samples. After resuspending the pellet in $1.5 \mathrm{ml}$ sterile distilled water, $1 \mathrm{ml}$ was used for mycobacterial culture and the remaining $0.5 \mathrm{ml}$ was stored at $4{ }^{\circ} \mathrm{C}$ (for no longer than 3 days) until the cell-disruption stage of the GTMD test was performed. In five cases, the samples were stored at $-20{ }^{\circ} \mathrm{C}$ (for less than 1 month) until testing.

Identification of cultured isolates. Culture isolates were identified using the GenoType Mycobacterium CM assay (Hain Lifescience), which allows identification of 15 different mycobacterial species (M. avium, Mycobacterium chelonae, Mycobacterium abscessus, Mycobacterium fortuitum, Mycobacterium gordonae, $M$. intracellulare, Mycobacterium scrofulaceum, Mycobacterium interjectum, M. kansasii, M. malmoense, Mycobacterium peregrinum, Mycobacterium marinum/ Mycobacterium ulcerans, Mycobacterium xenopi and MTBC). If identification was not possible with this assay, then the GenoType Mycobacterium AS test (Hain Lifescience) was used for the identification of additional mycobacterial species: Mycobacterium simiae, Mycobacterium mucogenicum, Mycobacterium goodii, Mycobacterium celatum, Mycobacterium smegmatis, Mycobacterium genavense, Mycobacterium lentiflavum, Mycobacterium heckeshornense, Mycobacterium szulgai/Mycobacterium intermedium, Mycobacterium phlei, Mycobacterium haemophilum, M. kansasii, Mycobacterium ulcerans, Mycobacterium gastri, Mycobacterium asiaticum and Mycobacterium shimoidei. Both tests were performed following the manufacturer's instructions as outlined briefly below. Liquid and solid cultures were considered negative if no growth was obtained after 6 or 8 weeks' incubation, respectively.

GTMD method. The complete procedure is divided into four stages. (i) Following heat lysis and sonication of cells, magnetic beads were used to capture the $23 \mathrm{~S}$ rRNA in a coupling reaction. (ii) After two washing steps, a nucleic acid sequence-based amplification reaction was carried out at $41{ }^{\circ} \mathrm{C}$ for $1 \mathrm{~h}$ to amplify sequences from the ssRNA template. (iii) The denatured amplified products were subsequently identified by reverse hybridization to immobilized gene probes. (iv) Colorimetric detection of the hybridized amplicons was carried out using streptavidin-conjugated alkaline phosphatase and the appropriate substrate.

Internal control RNA was included with every test sample to check for inhibitory substances. Positive and negative controls were included in each test run. The positive control contained M. avium RNA to assess the performance of the test and the negative control indicated whether sample-to-sample contamination had occurred. RNA contamination was limited by swabbing benches and equipment with bleach before use. To interpret the test results, a template sheet showing the positions of the lines on each strip (provided with the kit) was used. The assay time was approximately $5 \mathrm{~h}$, allowing the results to be generated in one working day.

\section{RESULTS AND DISCUSSION}

In this study, we evaluated the suitability of a commercially available GTMD assay (version 2.0) for the rapid identification of five mycobacterial species, including $\mathrm{MTBC}$, in smear-positive respiratory samples.
Microscopy was performed on 53/56 respiratory samples using auramine phenol staining. Microscopy was not performed on three sputum specimens that had been received as part of an external quality assurance programme. Twenty-two samples were found to contain few AFB $(+), 11$ contained moderate numbers of AFB $(++)$ and 20 contained many AFB $(+++)$.

In group $A, 17 / 20$ samples (85\%) in which RNA amplification was successful produced GTMD test results that concurred with the identification of the cultured isolates (Table 2). Eleven samples produced the hybridization pattern of MTBC and two showed that of $M$. malmoense. The four samples that failed to produce any hybridization signal to the five probes on the strips were shown by culture to contain other atypical mycobacteria (M. xenopi, M. fortuitum, M. simiae and M. celatum). These negative GTMD findings were in agreement with the culture results, as these mycobacterial species are not detectable by the molecular test. Three samples gave negative GTMD results although M. avium and M. kansasii were identified by specimen culture. Microscopic analysis showed that these specimens contained only small numbers of bacilli prior to being divided for decontamination and therefore there may have been too few bacilli for detection.

The conjugate control bands were visible in all cases and the amplification control bands showed a strong positive signal in all but three cases (specimens 1,13 and 19). For specimen 19 , the MTBC-specific band was also present, indicating that the isolation and amplification reactions had been performed correctly and the test result was valid. The other two sputum specimens found to contain $M$. tuberculosis by culture appeared to contain nucleic acid amplification inhibitors as shown by the reduction in or lack of positive signal from the internal amplification control band on the strips. Specimen 13 showed partial inhibition of RNA amplification, as the amplification control band was very faint but still visible. On the other hand, no control RNA amplicon was generated during amplification of sample 1 , as the conjugate control band was the only band present. Interestingly, another aliquot of sample 1 (specimen 3 ) had been stored at $-70{ }^{\circ} \mathrm{C}$ and was retested. This sample gave a positive hybridization signal for both controls and MTBC. We speculate that this stems from the removal of naturally occurring inhibitors of the amplification enzymes as a result of the freeze-thaw process. No further aliquot of the other sample was available for testing.

In group B, 14/17 GTMD test results (82\%) were concordant with the culture results (Table 3). Seven samples were found to contain MTBC, three samples contained M. kansasii and one contained M. malmoense. Two samples were culture-negative after 8 weeks' incubation and thus did not appear to contain viable mycobacteria. In addition, one smear-positive sample was found to contain $M$. intracellulare using the GTMD test, but the isolate failed to grow on culture after 8 weeks, indicating a false-negative culture result. Two samples produced growth 
Table 2. Comparison of GTMD results with culture identification for group A clinical specimens

AC, amplification control; NEG, negative result; + , few AFB seen upon direct microscopy; ++ , moderate numbers of AFB seen upon direct microscopy; +++ , many AFB seen upon direct microscopy.

\begin{tabular}{|c|c|c|c|c|c|}
\hline Study no. & Smear result & Frozen at $-70{ }^{\circ} \mathrm{C}$ & Storage time (months) & Culture ID & GTMD result \\
\hline 1 & +++ & No & 0 & M. tuberculosis & NEG (no AC band) \\
\hline 2 & ++ & No & 0 & M. simiae & NEG \\
\hline 3 & +++ & Yes & 1 & M. tuberculosis & MTBC \\
\hline 4 & +++ & Yes & 1 & M. tuberculosis & MTBC \\
\hline 5 & +++ & Yes & 1 & M. tuberculosis & МТBC \\
\hline 6 & + & Yes & 1 & M. tuberculosis & MTBC \\
\hline 7 & +++ & Yes & 2 & M. tuberculosis & MTBC \\
\hline 8 & ++ & Yes & 2 & M. tuberculosis & MTBC \\
\hline 9 & ++ & Yes & 2 & M. tuberculosis & MTBC \\
\hline 10 & + & Yes & 2 & M. malmoense & M. malmoense \\
\hline 11 & + & Yes & 2 & M. kansasii & NEG \\
\hline 12 & +++ & Yes & 2 & M. celatum & NEG \\
\hline 13 & + & Yes & 3 & M. tuberculosis & NEG (weak AC band) \\
\hline 14 & +++ & Yes & 3 & M. tuberculosis & МТВС \\
\hline 15 & + & Yes & 3 & M. tuberculosis & MTBC \\
\hline 16 & $+1-$ & Yes & 3 & M. xenopi & NEG \\
\hline 17 & +++ & Yes & 3 & M. fortuitum & NEG \\
\hline 18 & + & Yes & 4 & M. tuberculosis & MTBC \\
\hline 19 & +++ & Yes & 4 & M. tuberculosis & MTBC (no AC band) \\
\hline 20 & + & Yes & 4 & M. avium & NEG \\
\hline 21 & + & Yes & 5 & M. malmoense & M. malmoense \\
\hline 22 & + & Yes & 7 & M. avium & NEG \\
\hline
\end{tabular}

of $M$. avium but gave negative GTMD results. A further sample was found to contain M. kansasii using the GTMD test but contained M. abscessus only on culture. This sample had been taken from a patient with cystic fibrosis who had previous isolates of $M$. kansasii. It is probable that M. kansasii was also present in the sputum but was masked

Table 3. Comparison of GTMD results with culture identification for group B clinical specimens

NG, no growth after 8 weeks' incubation; NEG, negative result; smear results, see Table 2 for classification.

\begin{tabular}{|lccll|}
\hline Study no. & Smear result & Storage at $\mathbf{4}^{\circ} \mathbf{C}$ (months) & \multicolumn{1}{c|}{ Culture ID } & GTMD result \\
\hline 23 & + & 0 & M. tuberculosis & MTBC \\
24 & + & 0 & NG & M. intracellulare \\
25 & ++ & 0 & M. avium & NEG \\
26 & + & 0 & M. abscessus & M. kansasii \\
27 & ++ & 0 & M. tuberculosis & MTBC \\
28 & + & 0 & M. tuberculosis & MTBC \\
29 & + & 0 & NG & NEG \\
30 & + & 0 & M. tuberculosis & MTBC \\
31 & +++ & 0 & M. kansasii & M. kansasii \\
32 & + & 0 & M. tuberculosis & MTBC \\
33 & ++ & 0 & M. kansasii & M. $k a n s a s i i$ \\
34 & ++ & 0 & NG & NEG \\
35 & +++ & 0 & M. tuberculosis & MTBC \\
36 & + & 0 & M. avium & NEG \\
37 & +++ & 0 & M. tuberculosis & MTBC \\
38 & +++ & 0 & M. kansasii & M. kansasii \\
39 & +++ & 0 & M. malmoense & M. malmoense \\
\hline
\end{tabular}

${ }^{\star}$ Specimen from a patient with cystic fibrosis who had a previous $M$. kansasii isolate. It is probable that $M$. kansasii was masked by the rapidly growing $M$. abscessus on solid culture media. 
Table 4. Comparison of GTMD results with culture identification for group $\mathrm{C}$ clinical specimens

NG, no growth after 8 weeks' incubation; NEG, negative result; ND, not done; BAL, bronchoalveolar lavage; smear results, see Table 2 for classification.

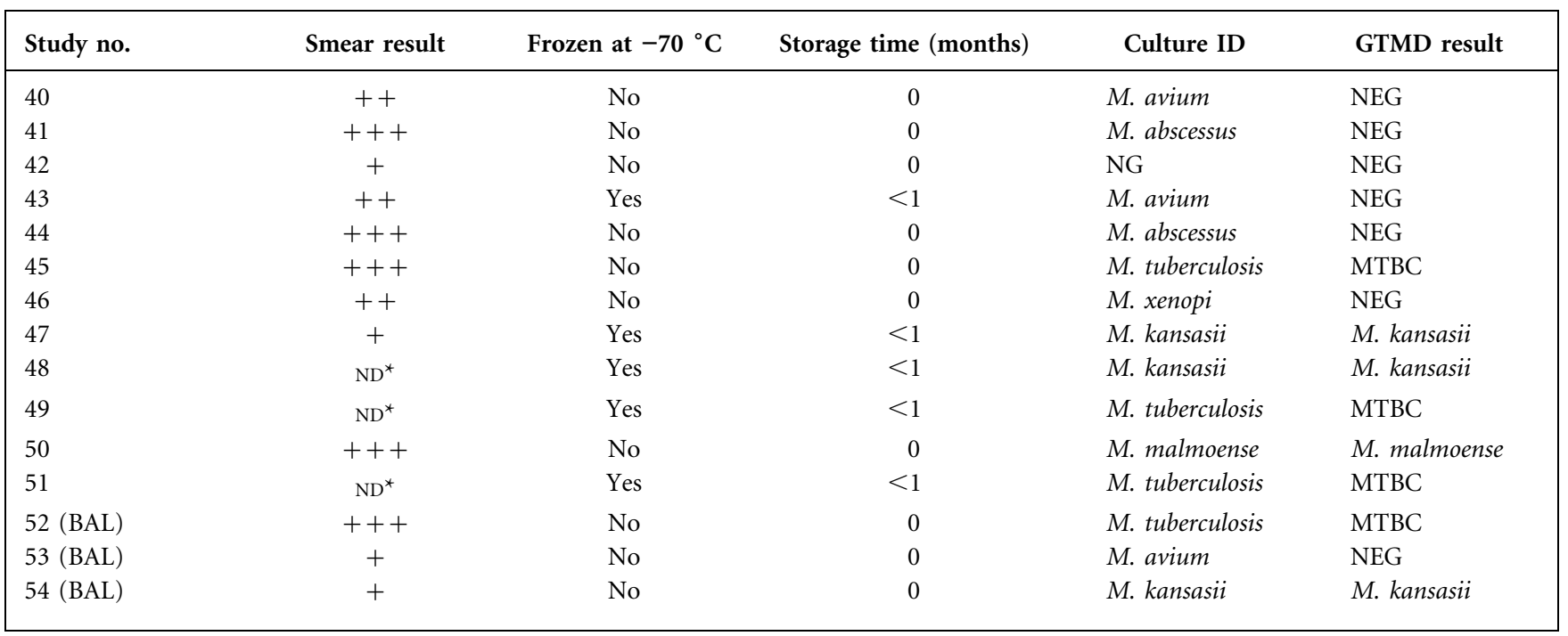

${ }^{\star}$ Quality control samples for direct detection - microscopy was not done.

by the rapidly growing $M$. abscessus on the solid culture medium.

In group C, 12/15 GTMD test results (80\%) were concordant with the culture results (Table 4). Four samples contained MTBC, three samples contained M. kansasii, one contained $M$. malmoense and three contained other atypical mycobacteria (two M. abscessus and one $M$. xenopi). One sample was negative by both culture and the molecular test. Three samples gave false-negative GTMD results, as M. avium was obtained by culture.

Overall, 18 samples produced hybridization signals only for the control bands, suggesting that no mycobacteria detectable by the assay were present. Three of these samples were culture-negative after 8 weeks' incubation and did not contain viable bacilli, despite AFB being seen on fluorescence microscopy. One of the patients was known to have returned from abroad, where TB had been partially treated using an unknown treatment regime. The drug treatment status of the other two patients was unknown. Seven samples contained atypical mycobacteria not detectable using the GTMD assay (one each containing M. fortuitum, M. simiae and M. celatum and two each containing $M$. xenopi and M. abscessus) and eight samples gave apparent false-negative GTMD results, M. avium and M. kansasii having been identified by specimen culture.

Two samples were omitted from the analysis due to partial inhibition or lack of amplification of the internal control RNA. In 52 samples in which amplification occurred successfully, $43(82.6 \%)$ produced final GTMD results that were confirmed by culture (Table 5). All cases of culturepositive MTBC were detected by the molecular test.

Table 5. Summary of GTMD test results for all 54 specimens tested

\begin{tabular}{|c|c|c|c|c|c|c|c|c|c|c|}
\hline Group & $n$ & MTBC & M. avium & M. malmoense & M. kansasii & M. intracellulare & $\begin{array}{l}\text { Other atypical } \\
\text { mycobacteria }\end{array}$ & False-negative & $\begin{array}{c}\text { No } \\
\text { growth }\end{array}$ & Inhibition \\
\hline A & 22 & $11^{*}$ & 0 & 2 & 0 & 0 & 4 & $\begin{array}{c}3 \text { (2 M. avium, } \\
\text { M. kansasii) }\end{array}$ & 0 & 2 \\
\hline B & 17 & 7 & 0 & 1 & $4 \dagger$ & $1 \neq$ & 0 & 2 (M. avium) & 2 & 0 \\
\hline $\mathrm{C}$ & 15 & 4 & 0 & 1 & 3 & 0 & 3 & 3 (M. avium) & 1 & 0 \\
\hline Total & 54 & 22 & 0 & 4 & 7 & 1 & 7 & 8 & $3 \S$ & 2 \\
\hline
\end{tabular}

${ }^{\star}$ In one specimen, amplification of the control RNA was suppressed by amplification of the specific product.

†Contained M. abscessus on solid culture medium, which probably masked M. kansasii. Thus the GTMD test result could not be confirmed.

$\ddagger$ False-negative culture result as no growth was visible at 8 weeks but $M$. intracellulare RNA was detected.

$\S$ No RNA amplified using the GTMD test and no growth observed using solid culture media. It is probable that these samples contained no viable mycobacteria. 
Twenty-two samples contained MTBC, seven had $M$. kansasii, four had M. malmoense, one had M. intracellulare, seven contained other atypical mycobacteria not detectable using the GTMD assay and three specimens contained no viable mycobacteria. Results for eight of the remaining nine samples indicated false-negative GTMD results, as $M$. avium $(n=7)$ and M. kansasii $(n=1)$ were isolated upon specimen culture. The molecular test indicated $M$. avium from one smear-positive sample that was culture-negative. Although the number of samples tested was small, the decontamination method used did not appear to have any effect on assay performance, with $82 \%$ of NALC-treated specimens and $80 \%$ of $\mathrm{NaOH}$-treated specimens producing concordant GTMD and culture identification results.

The GTMD assay showed $100 \%$ concordance with culture for the identification of all cases of MTBC and $M$. malmoense infection. The test was specific, with no instances of cross-reaction with specimens containing other mycobacterial species. We would, however, recommend further investigation of specimens from patients with a negative GTMD test result due to the number of falsenegative results obtained for patients with M. avium infection. It was interesting to note that four of the seven false-negative $M$. avium GTMD results and a false-negative $M$. kansasii result were obtained from samples collected from patients who had been smear-positive for some time and had commenced antimicrobial therapy. Thus the lack of nucleic acid amplification from these $M$. avium isolates may not have been due to a strain-specific characteristic but may have been the result of the inhibitory effects of anti-mycobacterial drugs on RNA despite viability remaining intact. This may have been the situation for three additional samples, but treatment information for these patients was not available so this could not be confirmed.

For optimal assay performance, we recommend that the entire specimen volume is used for processing, as dividing the specimen into two equal volumes prior to NALC decontamination (as for group A) may have accounted for three of the seven false-negative GTMD test results (two $M$. avium and one $M$. kansasii). Microscopic examination showed few AFB (+) to be present in these specimens, which were divided further prior to decontamination. This may have resulted in too few bacilli in the aliquot tested to be detected by the GTMD assay. From a laboratory perspective, the processing protocols used for group B and $\mathrm{C}$ specimens were found to be more appropriate, as the entire specimen was decontaminated using either NALC/ $3 \% \mathrm{NaOH}$ (group B) or $4 \% \mathrm{NaOH}$ (group C) without any adverse effect on the GTMD results. It was possible to detect mycobacterial RNA after storage of specimens at $-70{ }^{\circ} \mathrm{C}$ for 5 months, but freezing specimens for as long as 7 months is not recommended as RNA degradation may occur.

The GTMD assay is easy to perform and can be completed in one working day. Although it takes longer than some of the other commercially available direct nucleic acid amplification methods, it does provide rapid detection of four additional mycobacterial species rather than MTBC alone. Interpretation of GTMD test results compared with some other commercial nucleic acid assays is also made easier for the user by the inclusion of an internal amplification control to monitor the presence of inhibitors that interfere with nucleic acid amplification. Such inhibitors can lead to false-negative results in $3-15 \%$ of clinical samples (Forbes \& Hicks, 1996). In our study, RNA amplification was successful in most cases and thus nucleic acid amplification inhibitors did not appear to be a major problem.

It is difficult to compare the results from this work directly with the large number of other reports published using CDATs. The literature reflects the fact that groups have assessed different CDATs using different specimen types and assessed their outcome using different criteria. Compared with culture, our results show the sensitivity, specificity, and positive and negative predictive values for the detection of MTBC, M. avium, M. intracellulare, $M$. kansasii and M. malmoense using GTMD to be 80.5, 75, 97 and $27.3 \%$, respectively. These figures differ from the results of 92, 100, 100 and $77 \%$ from a larger study by Franco-Álvarez de Luna et al. (2006) primarily due to the larger proportion of false-negative GTMD results obtained in our study (17 vs $5.9 \%$ ). Nonetheless, we are encouraged by the results from this work and believe that a GTMDpositive result has the potential to significantly improve patient care and public health by providing rapid and specific information to clinicians about the presence of MTBC and some other common atypical mycobacteria in smear-positive samples. Further work is required to assess the sensitivity of the assay for non-respiratory samples and smear-negative samples, although some success has been reported with identification of MTBC in biopsy specimens, bronchial aspirates, an abscess and a faecal specimen (Franco-Álvarez de Luna et al., 2006). In our hands, the GTMD test has identified $M$. tuberculosis in one tissue specimen. The isolate had failed to grow on solid culture, despite prolonged incubation. From a laboratory perspective, a new version (version 4.0) of the GTMD assay includes a modified protocol to reduce the amount of specimen manipulation required during the RNA isolation stage and to improve sensitivity. Rapid diagnosis and differentiation between MTBC and NTM allows the appropriate drug susceptibility panel to be tested as soon as the isolate has grown. Whilst this can save time in the laboratory, far greater savings are likely across public health due to improved patient care and infection control measures as a result of a speedier identification service.

\section{ACKNOWLEDGEMENTS}

The authors would like to thank the staff in both laboratories for their help with processing and decontamination of specimens used in this study. 


\section{REFERENCES}

Falkinham, J. O., III (1996). Epidemiology of infection by nontuberculous mycobacteria. Clin Microbiol Rev 9, 177-215.

Forbes, B. A. \& Hicks, K. E. (1996). Substances interfering with direct detection of Mycobacterium tuberculosis in clinical specimens by PCR: effects of bovine serum albumin. J Clin Microbiol 34, 2125-2128.

Franco-Álvarez de Luna, F., Ruiz, P., Gutiérrez, J. \& Casal, M. (2006). Evaluation of the GenoType Mycobacteria Direct assay for detection of Mycobacterium tuberculosis complex and four atypical mycobacterial species in clinical samples. J Clin Microbiol 44, 3025-3027.

Health Protection Agency Centre for Infections (2007). Tuberculosis in the UK: annual report on tuberculosis surveillance and control in the UK 2007. http://www.hpa.org.uk/publications/ 2007/TB/TB_annual_report.pdf (last accessed 04 January 2008).

National Collaborating Centre for Chronic Conditions (2006). Tuberculosis: Clinical Diagnosis and Management of Tuberculosis, and Measures for its Prevention and Control. London: Royal College of Physicians.

Piersimoni, C. \& Scarparo, C. (2003). Relevance of commercial amplification methods for direct detection of Mycobacterium tuberculosis complex in clinical samples. J Clin Microbiol 41, 5355-5365.

Shakir, E., Johnston, F. \& McMenamin, J. (2007). Enhanced surveillance of mycobacterial infections (ESMI) in Scotland: annual report for Scotland for the years 2004-2006. HPS Wkly Rep 41, 374-380. 